\title{
Comprehensive Study on the Electron Temperature and Electron Density of Laser-Induced Mg Plasma
}

\author{
Hongbing $\mathrm{Y}^{1}$, Asamoah $\mathrm{E}^{1 *}$, Jiawei $\mathrm{C}^{1}$, Dongqing $\mathrm{Y}^{\mathbf{2}}$ and Fengxiao $\mathrm{Y}^{1}$ \\ ${ }^{1}$ School of Mechanical Engineering, Jiangsu University, Zhenjiang, Jiangsu, China \\ ${ }^{2}$ Department of Mathematics and Physics, Huaihai Institute of Technology, Lianyungang, Jiangsu, China
}

\begin{abstract}
In this study, we shall report the spectroscopic studies of the laser induced-Mg plasma in the atmospheric air by a Q-switched Nd-YAG laser operating at its fundamental wavelength of $1062 \mathrm{~nm}$. The plasma was evaluated as a function of distance along the plasma expansion from 0.5-5.5 mm. The electron temperature and the electron number density were determined from the Boltzmann's plot method and the Stark broadening methods respectively. From our investigations the electron temperature was evaluated to be in the range of $17556-9785 \mathrm{~K}$ for the laser energy of $500 \mathrm{~mJ}$ and $17341-8946 \mathrm{~K}$ with laser energy of $450 \mathrm{~mJ}$. The electron number density was deduced to be from $1.46 \times 10^{17}-1.89 \times 10^{17} \mathrm{~cm}^{-3}$ and $1.29 \times 10^{17}-1.78 \times 10^{17} \mathrm{~cm}^{-3}$ respectively. From our evaluations, we observed that the electron temperature and number density decrease along the distance of the plasma expansion, and also increases with increasing laser energy.
\end{abstract}

Keywords: Laser induced plasma; Plasma spectroscopy; Electron temperature; Emission spectroscopy; Mg spectra; Electron density

\section{Introduction}

As a spectroscopic technique, laser induced breakdown spectroscopy (LIBS) have attracted numerous attention in the scientific community for the past decades, due to its potential application in fields such as material processing, biomedical, military, industrial analysis, pharmaceutical studies, and environmental monitoring [1-6]. Laser induced breakdown spectroscopy (LIBS) which is sometimes referred to as laser-induced plasma spectroscopy (LIPS) uses the radiations produced from the plasma as a result of the interaction of the laser with the target material, to analyze qualitatively and quantitatively the elemental composition of the target sample [7]. LIBS hold numerous advantages over other conventional atomic emission spectroscopy techniques: LIBS is applicable to both conducting and non-conducting target sample analysis, and it also requires little or no preparation of sample [8-11]. LIBS employs a high power energy laser pulse which is focused onto a target material by a focusing lens to ablate the target surface to generate a plasma plume $[12,13]$. The light emitted is collected, through a fiber optic cable into the spectrometer. The light is then analyzed by the spectrometer, which is used to characterize and investigate the sample composition, electron temperature and number density.

The laser-target interaction process can be classified into; the evaporation of the surface (ablation of the target material), plasma formation of the ablated material, plasma expansion, and plasma radiation and cooling. The analytical performance of laser produced plasmas is influenced by several factors such as: (i) the material properties; (ii) laser parameters. The properties of plasma that affects light emitting are the electron density, electron number density and electron temperature of the emitting species. Therefore the knowledge of plasma temperature and electron density is necessary to understand the physical processes involved $[14,15]$.

In our previous work [16], we investigated on the influence of laser energy on the electron temperature and we found that, the electron temperature increases rapidly with increasing laser energy. Several research papers have been reported on the electron temperature and electron density of a plasma plum. Krähling et al. [17] investigated on the electron temperature and number density of a liquid electrode dielectric barrier discharge, LE-DBD from the Stark broadening of three strontium lines. They also studied the $\mathrm{OH}(\mathrm{A})$ rotational distribution, and found that it exhibited a non-Boltzmann characteristic with a superposition of two Boltzmann distributions.

Mahmood et al. [18] also investigated on the electron temperature and density of a neon in a hollow cathode discharge lamb, using Boltzmann's plot and Stark broadening respectively. They also studied the behavior of the optogalvanic signal as a function of laser energy.

In this study, we shall report on the investigation of laser-induced $\mathrm{Mg}$ plasma produced by $1064 \mathrm{~nm}$ Nd:YAG laser in the atmospheric air. The spatial evolution of the emission has been resolved along the distance of the plasma expansion. We have studied the electron temperature of the $\mathrm{Mg}$ plasma from the Boltzmann plot method using the relative intensities ratio of the $\mathrm{Mg}$ spectral lines. We further, studied the electron number density of the $\mathrm{Mg}$ plasma from the Stark broadening method, and the temporal evolution of the electron temperature and number density have also been resolved.

\section{Experimental Setup and Procedure}

The schematic diagram of the experimental setup of a laser induced breakdown spectroscopy is depicted in the Figure 1. A Q-switched NdYAG laser operating at its fundamental wavelength of $1064 \mathrm{~nm}$ having a pulse duration of $10 \mathrm{~ns}$ and a repetition frequency of $1 \mathrm{~Hz}$ with a laser energies of $400 \mathrm{~mJ}, 450 \mathrm{~mJ}$ and $500 \mathrm{~mJ}$, measured by a pyrometric detector was used to generate the plasma.

*Corresponding author: Asamoah E, School of Mechanical Engineering, Jiangsu University, Zhenjiang, Jiangsu, China, Tel: +86 5118878 0011; E-mail: asamoah_grace@hotmail.com

Received March 25, 2018; Accepted April 18, 2018; Published April 25, 2018

Citation: Hongbing Y, Asamoah E, Jiawei C, Dongqing Y, Fengxiao Y (2018) Comprehensive Study on the Electron Temperature and Electron Density of Laser-Induced Mg Plasma. J Laser Opt Photonics 5: 181. doi: 10.4172/2469 410X.100018

Copyright: (c) 2018 Hongbing Y, et al. This is an open-access article distributed under the terms of the Creative Commons Attribution License, which permits unrestricted use, distribution, and reproduction in any medium, provided the original author and source are credited. 


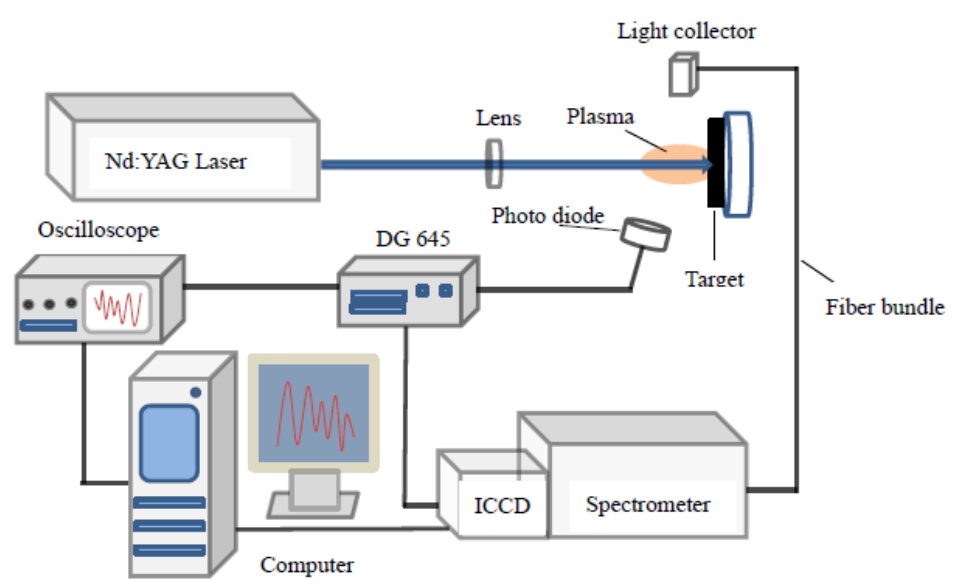

Figure 1: Schematic diagram of the experimental set-up for laser induced breakdown spectroscopy.

A quartz lens with $150 \mathrm{~mm}$ focal length was used to focus the laser on the target surface. The target was a certified $99.99 \%$ magnesium plate, which was mounted on a three-dimensional xyz-stage. The distance between the target and the laser was set slightly shorter than the focal length of the lens in order to avoid any breakdown of the ambient air in front of the target surface. The data acquisition was done by polishing the surface of the target material before it was exposed to the focused laser beam. The spot area of the laser beam was $\sim 1 \mathrm{~mm}^{2}$ on the target surface.

The light emitted from the plasma was collected and analyzed by an optical fiber bundle in conjunction with a Mechelle spectrometer equipped with a getable intensified charged-coupled device (ICCD) camera $(1024 \times 1024)$ pixels istar from Andor Technology). The ICCD was triggered by a digital generator (Stanford Research System, model DG645) connected to an oscilloscope (YOKOGAWA, digital oscilloscope DL9140, Japan). Throughout the experiment the gate width was kept at $1 \mu$ s and the delay time was varied by means of a delay generator, which was monitored by signals collected by the photodiode to the digital oscilloscope.

The target was translated after 15 laser shots, and to ensure that the signal to noise ratio was increased. The experiment was done at a room temperature in air and atmospheric pressure.

\section{Results and Discussion}

\section{Plasma emission and spectral analyses}

The interaction of a high-power laser with a sample of magnesium target generated a plasma plume. The light from the plasmas were collected for analyses. The emission spectra of the plasma was recorded at different distances along the direction of the plasma expansion with a plasma energies of $400 \mathrm{~mJ}, 450 \mathrm{~mJ}$ and $500 \mathrm{~mJ}$ in air at atmospheric pressure as depicted in the Figure 2a-c plasma spectra induced by the laser consists of a strong continuum and ionic lines of the components of the target sample. The plasma expansion rapidly drops out at larger distance from the target surface [19].

In order to avoid inhomogeneity and time integration, which may as a result affect the spectral line profile. Care was taken in selecting- the time between the laser shot and the data acquisition- the delay time and the gate width. Several delay times were used for the plasma diagnosis which ranges from $1 \mu \mathrm{s}-16 \mu \mathrm{s}$. The selected delay times and gate width was suitable to enhance the signal-to-background ratio.
We assumed that the plasma was quasi-stationary for the delay times chosen for our experiment.

From the Mg emission spectra obtained the line intensity rapidly decreases with increasing delay time, as depicted in Figure 3. This is as a result of the radiative recombination between the electrons and ions, and the Bremsstrahlung emission.

\section{Determination of electron temperature}

The spectroscopic performance of the plasma can be analyzed by the parameters such as the electron density, electron number density and temperature of the species. If thermodynamic equilibrium exists, then the properties of the plasma such as speed distributions of the particles and the population of the energy levels can be explained through the temperature. The electron temperature is determined from the relative intensities of the $\mathrm{Mg}$ I lines utilizing the Boltzmann plot method. This method is applied by assuming that the excitation temperature and the kinetic temperature are equal. This requires the plasma to be in the state of local thermodynamic equilibrium LTE. For the Mg plasma lines used, the validity of the existence of the local thermodynamic equilibrium was satisfied for longer delay times [20,21]. In most papers the McWhirter criterion has been widely used to justify the existence of LTE. Which can be mathematically represented as [22]?

$$
N_{e} \geq 1.6 \times 10^{12} T^{1 / 2}\left(\Delta E_{m n}\right)^{3}
$$

Where $T$ is the plasma temperature (K), $\Delta E_{m n}$ higher energy difference $(\mathrm{eV})$ of the $\mathrm{Mg}$ lines used and $N_{e}$ is the electron density $\left(\mathrm{cm}^{-3}\right)$.

Recent publications have shown that McWhirter criterion is essential but insufficient justification technique for local thermodynamic equilibrium to exist [23].

From Boltzmann's distribution law, the population of the energy levels of the species at a given position can be mathematically expressed as:

$$
\frac{N_{j}^{z}}{N^{z}}=\frac{\mathrm{g}_{\mathrm{j}}^{\mathrm{z}} e \frac{-E_{j}^{z}}{k T}}{Q^{z}(T)}
$$

where the superscript $\mathrm{z}$ represents the ionization of the species $(\mathrm{z}=0)$ for the neutral ionized atoms and $\mathrm{z}=1$ for singly ionized atoms), $N_{j}^{z}$ and $\mathrm{g}_{\mathrm{j}}^{\mathrm{z}}$ are the population of the energy levels $\left(\mathrm{m}^{-3}\right)$ and degeneracy of the upper energy levels respectively. $E_{j}^{z}$ is the energy $(\mathrm{eV}), N^{z}$ is the number density of the species $\left(\mathrm{m}^{-3}\right), k$ is the Boltzmann's constant $\left(\mathrm{eVK}^{-1}\right), \mathrm{Q}^{Z}(\mathrm{~T})$ 
(a)

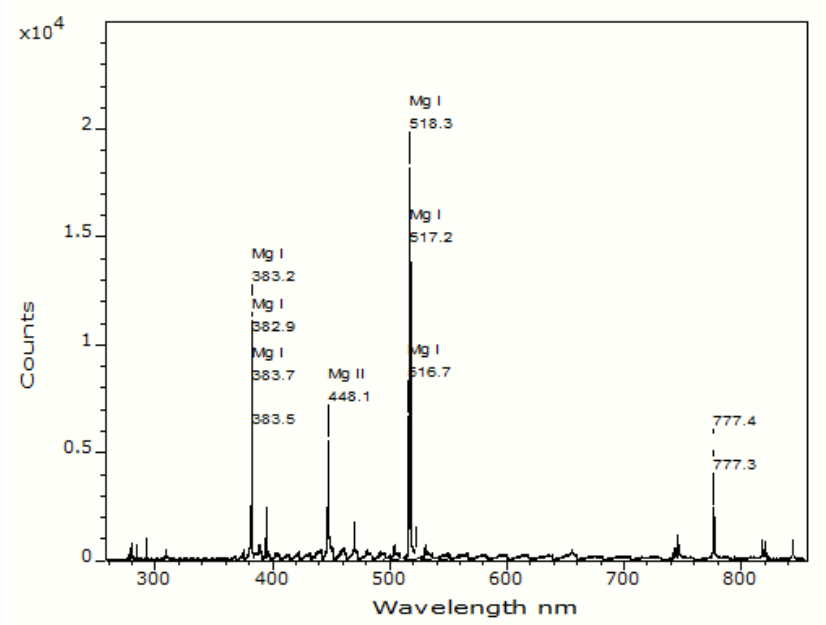

(c)

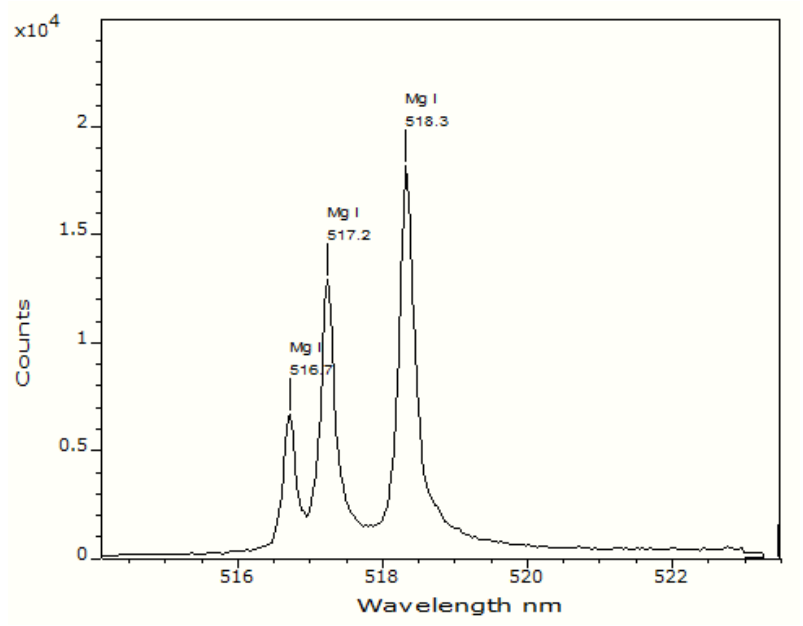

(b)

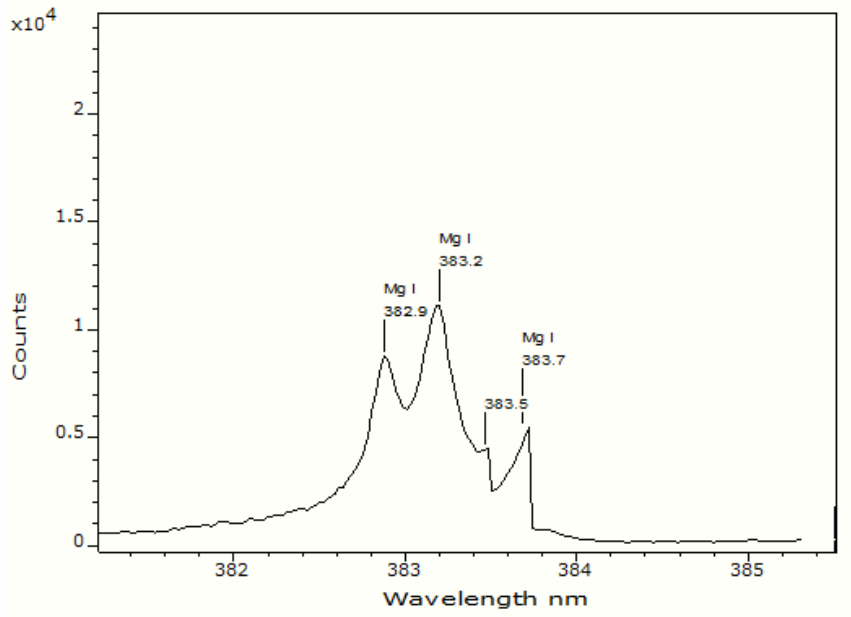

Figure 2: (a) The emission spectrum generated by a $1064 \mathrm{~nm}$ laser at a distance of $1 \mathrm{~mm}$ from the target surface, with a delay time of $1 \mu$ s and laser energy of 500 $\mathrm{mJ}$ (b) Spectral region from $382 \mathrm{~nm}-385 \mathrm{~nm}$ (c) Spectral region from $516 \mathrm{~nm}-520 \mathrm{~nm}$.

is the partition function and $T$ is the electron temperature. From eqn. (2), the emissivity $\varepsilon_{\mathrm{ji}}^{\mathrm{z}}\left(\mathrm{Wm}^{-3}\right)$ of a given species at a particular transition can be represented as

$$
\varepsilon_{\mathrm{ji}}^{\mathrm{z}}=\frac{h c}{\lambda_{j i}} A_{j i}^{z} \frac{N^{z}}{Q^{z}(T)} \mathrm{g}_{\mathrm{j}}^{\mathrm{z}} e \frac{-E_{j}^{z}}{k T}
$$

where $A_{j} i^{z}$ denotes the transition probability $\left(\mathrm{s}^{-1}\right), \lambda_{j i}$ represents the transition wavelength $(\mathrm{m}), h$ is the Planck's constant (Js) and $c$ is the speed of light $\mathrm{ms}^{-1}$ Taking the natural logarithm of the expression of the emissivity in equation (3) $[14,18,24,25]$ :

$$
\mathrm{n}\left(\frac{\varepsilon_{\mathrm{ji}}^{\mathrm{z}} \lambda_{j i}}{A_{j i}^{z} \mathrm{~g}_{\mathrm{j}}^{z}}\right)=-\frac{1}{k T} E_{j}^{z}+\ln \left(\frac{h c N^{z}}{Q(T)}\right)
$$

By using the wavelength of the $383.2 \mathrm{~nm}, 470.3 \mathrm{~nm}$ and $518.4 \mathrm{~nm}$ and the data of the following parameters $A_{j} i^{z}, E_{j}^{z}$ and $\mathrm{g}_{\mathrm{j}}^{z}$ of $\mathrm{MgI}$ lines retrieved from the database of the NIST [26], a linear plot can be deduced from equation (4). Plotting the magnitude of the left hand side of the equation to the right hand side of the equation gives a linear

\begin{tabular}{|c|c|c|c|}
\hline $\boldsymbol{\lambda}_{\mathrm{jij}}(\mathbf{n m})$ & $\mathbf{E}_{\mathbf{j}}(\mathbf{e V})$ & $\mathbf{A}_{\mathbf{j i j}}\left(\mathbf{1 0}^{\mathbf{8}} \mathbf{s}^{\mathbf{-}}\right)$ & $\mathbf{g}_{\mathbf{j}}$ \\
\hline 383.0 & 5.95 & 0.90 & 3 \\
\hline 383.2 & 5.95 & 1.21 & 5 \\
\hline 470.3 & 6.98 & 0.22 & 5 \\
\hline 516.8 & 5.11 & 0.11 & 3 \\
\hline 517.3 & 5.11 & 0.34 & 3 \\
\hline 518.4 & 5.11 & 0.56 & 3 \\
\hline 448.1 & 11.63 & 2.33 & 8 \\
\hline
\end{tabular}

Table 1: The spectroscopic data of the Mgl and Mgll spectral lines retrieved from the database of NIST for the determination of the electron temperature.

graph of the slope equal to $-1 / K T$. The temperatures are then obtained through calculation from the value of the slopes of the Boltzmann's plot (Table 1).

From the Boltzmann's plot method the highest electron temperature were recorded at $17556 \mathrm{~K}$ with a delay time of $1 \mu \mathrm{s}$, and laser energy of $500 \mathrm{~mJ}$. At the same delay time and pulse repetition rate, an electron temperature of $17341 \mathrm{~K}$ and $17085 \mathrm{~K}$ were obtained for the energies of $450 \mathrm{~mJ}$ and $400 \mathrm{~mJ}$ respectively as depicted if Figure 4. Decreasing the pulse repetition frequency to $1 \mathrm{~Hz}$ and a delay time $1 \mu \mathrm{s}$, the electron 


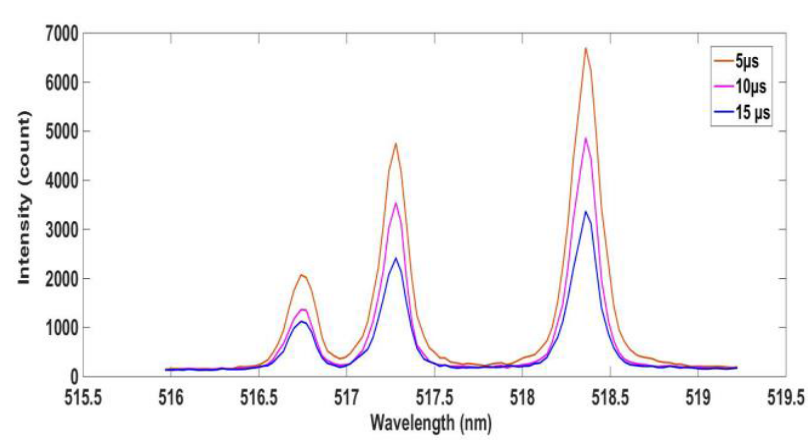

Figure 3: Time-resolved emission spectra of a laser-induced Mg plasma at a delay time of $5 \mu \mathrm{s}, 10 \mu \mathrm{s}$ and $15 \mu \mathrm{s}$, in the spectral region of $515.97-519.22 \mathrm{~nm}$.

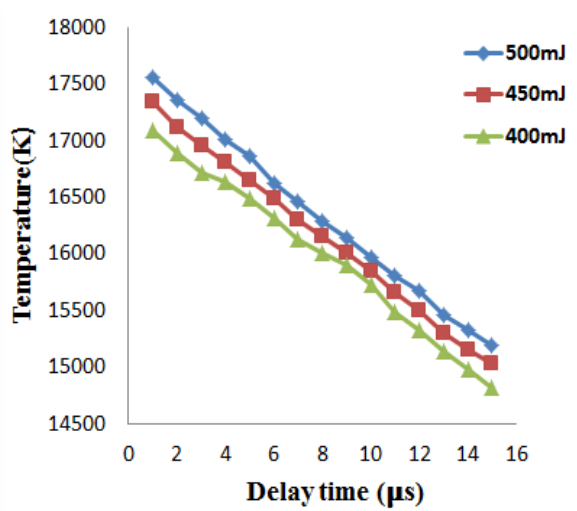

Figure 4: Temporal evolution of the electron temperature of the magnesium plasma, with a pulse repetition frequency of $5 \mathrm{~Hz}$, delay time of $1 \mu \mathrm{s}$ and a laser energy of $500 \mathrm{~mJ}$.

temperature dropped to $17350 \mathrm{~K}, 17121 \mathrm{~K}$ and 16891 with laser energies of $500 \mathrm{~mJ}, 450 \mathrm{~mJ}$ and $400 \mathrm{~mJ}$ respectively, as shown in Figure 5. A plot of the characteristic intensities of the $\mathrm{Mg}$ spectra versus the delay times in Figure 6, depicted a rapid decrease of the spectra intensities with increasing delay time.

We observed the spatial behavior of the emission spectra of the $\mathrm{Mg}$ plasma recorded at several distances along the direction of the expansion of the plasma plum from 0.5-5.5 $\mathrm{mm}$. The emitted electrons were established to be highly dense closer to the target surface. A graph of the electron temperature against the distance along the plasma expansion was depicted in Figure $7(a-b)$. When the target material was exposed to the laser energy at a distance close to the target surface, it constantly absorbs radiation during the exposure time of the pulse. This caused the electron to attain higher temperature. The higher value of the temperature closer to the surface of the magnesium target was as a result of the inverse Bremsstrahlung absorption and the rapid drop in temperature at a distance away from the magnesium target surface was also attributed to the conversion of the thermal energy into kinetic energy.

\section{Electron density of the plasma}

In this section, we report the measurements of the electron density for the Mg plasmas. The electron number density can be evaluated from the line broadening. The primary line broadening of the $\mathrm{Mg}$ lines are the Stark broadening, which is as a result of the collision of charged species. The Experimental line profile was fitted to a Lorentzian fit as depicted

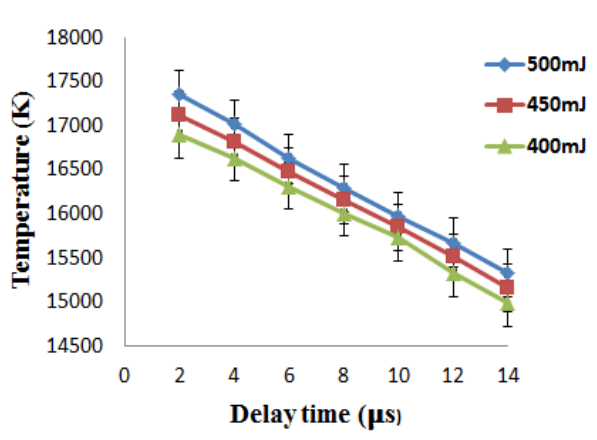

Figure 5: Temporal evolution of the electron temperature of the magnesium plasma, with pulse repetition frequency of $1 \mathrm{~Hz}$ and laser energy of $500 \mathrm{~mJ}$.

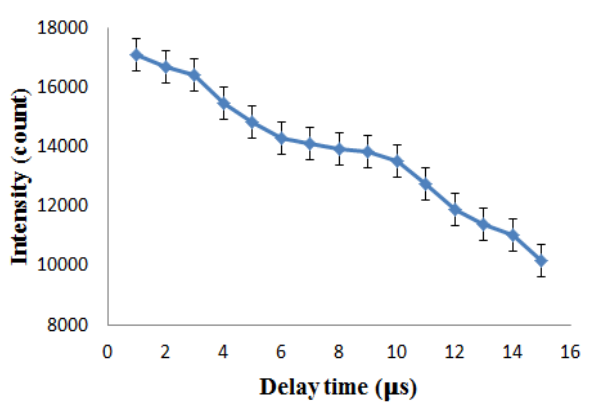

Figure 6: A plot of the characteristic intensities of the Mg spectra versus the delay time, with laser energy of $500 \mathrm{~mJ}$ and a pulse repetition rate of $1 \mathrm{~Hz}$.

(a)

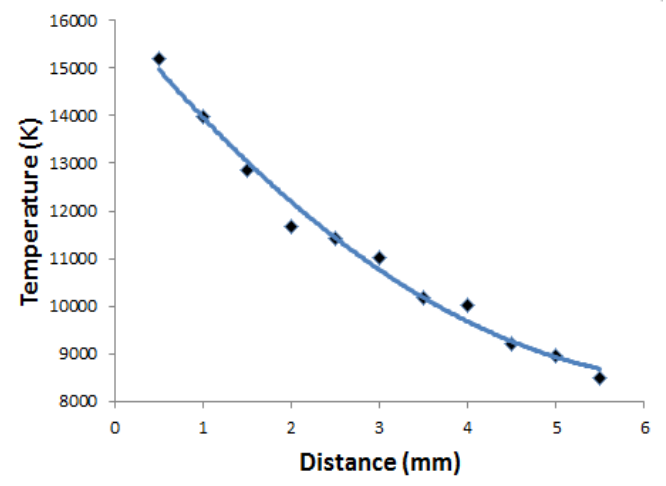

(b)

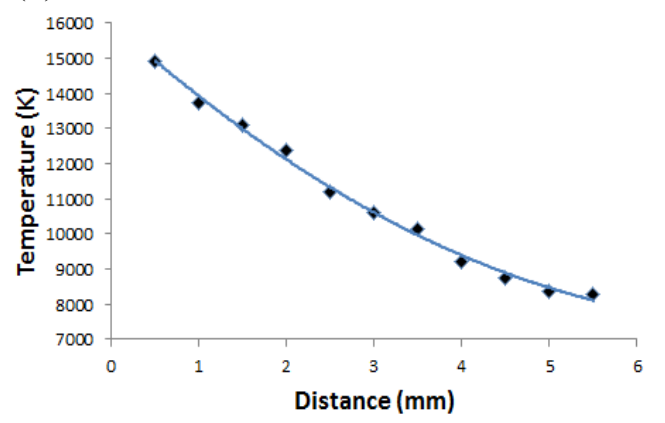

Figure 7: A graph of electron temperature against the distance along the plasma expansion. (a) with a delay time of $5 \mu \mathrm{s}$ and a laser energy of $500 \mathrm{~mJ}$ (b) with a delay time of $5 \mu \mathrm{s}$ and a laser energy of $450 \mathrm{~mJ}$. 
in Figure 8. From Maxwell distribution law, the half width FWHM for the Doppler broadening can be calculated from the expression [27,28];

$$
\Delta \lambda_{1 / 2}=2 \lambda \sqrt{2 k T \ln 2 / m c^{2}}
$$

Where $\lambda$ is the wavelength $(\mathrm{m}), \mathrm{T}$ is the absolute temperature $(\mathrm{K})$, $\mathrm{k}$ is the Boltzmann constant $\left(\mathrm{JK}^{-1}\right), \mathrm{m}$ is the represent the atomic mass $(\mathrm{kg})$ and $\mathrm{c}$ denotes the speed of light $\mathrm{ms}^{-1}$.

The half width FWHM of the stark broadening line can be related to the electron number density by the equation

$$
\Delta \lambda_{1 / 2}(n m)=2 \omega\left(\frac{N_{\mathrm{e}}}{10^{16}}\right)+3.5 \mathrm{~A} \times\left(\frac{N_{\mathrm{e}}}{10^{16}}\right)^{5 / 4}\left[1-\frac{3}{4} N_{D}^{-1 / 3}\right] \omega
$$

Where the ion broadening parameter is $\mathrm{A}(\mathrm{nm}), \omega(\mathrm{nm})$ is the electron impact width parameter, $\mathrm{N}_{\mathrm{D}}$ is the number of particles in the Debye sphere and $\mathrm{N}_{\mathrm{e}}\left(\mathrm{cm}^{-3}\right)$ is the electron number density. Since the broadening is mainly due to electron contribution, the ionic broadening can therefore be neglected. Hence the eqn. (6)

$$
\Delta \lambda_{1 / 2}(n m)=2 \omega\left(\frac{N_{\mathrm{e}}}{10^{16}}\right)
$$

The electron number density was determined to be in the range of $1.29 \times 10^{17}-1.78 \times 10^{17} \mathrm{~cm}^{-3}$ and $1.46 \times 10^{17}-1.89 \times 10^{17} \mathrm{~cm}^{-3}$ with an electron temperature ranges of $17341-8946 \mathrm{~K}$ and $17556-9785 \mathrm{~K}$ respectively. Figures $8-10$, depicts the temporal evolution of the electron number density with laser energies of $450 \mathrm{~mJ}$ and $500 \mathrm{~mJ}$ respectively. It was observed from the figures that the electron number density decreases slowly with the increase in the delay times.

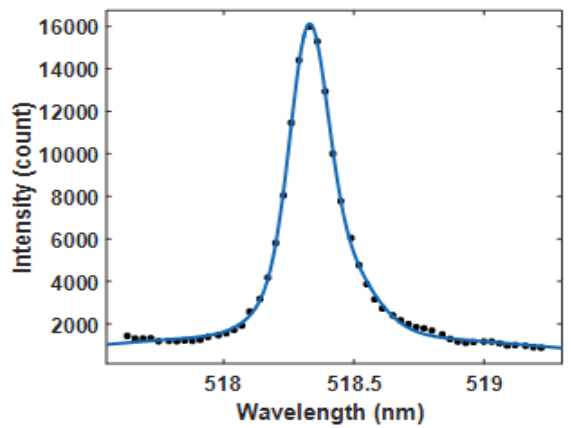

Figure 8: The Lorentz fit of the Mg spectral line at a delay time of $2 \mu$ s at laser energy of $450 \mathrm{~mJ}$.

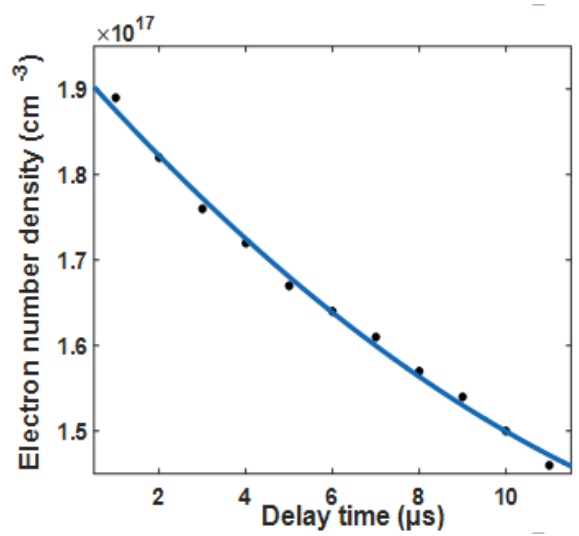

Figure 9: Temporal evolution of the electron number density of the Mg plasma with laser energy of $500 \mathrm{~mJ}$.

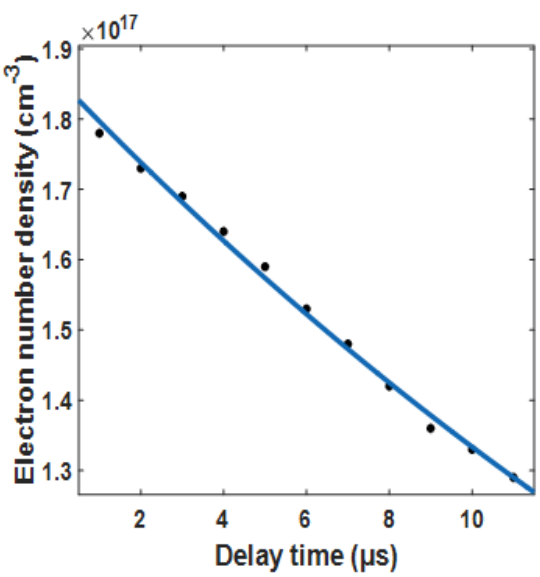

Figure 10: Temporal evolution of the electron number density of the $\mathrm{Mg}$ plasma at a delay time of $450 \mathrm{~mJ}$.

\section{Conclusion}

The spectroscopic performance of the Mg plasma induced by a Q-switch Nd-YAG laser was investigated. A study of the distance along the plasma expansion was also conducted from $0.5-5.5 \mathrm{~mm}$. The electron number density and the electron temperature were calculated from the Stark Broadening and the Boltzmann's plot method respectively. At a delay time of $1 \mu \mathrm{s}$ and laser energy of $500 \mathrm{~mJ}$, the highest temperature of $17556 \mathrm{~K}$ was obtained from the calculations. With further increase in the delay time from $1 \mu$ s to $16 \mu \mathrm{s}$, the electron temperature rapidly decreased to $9785 \mathrm{~K}$. Temperatures of 17341-8946 K was also recorded for the laser energy of $450 \mathrm{~mJ}$. The electron number density was also determined to be in the range of $1.46 \times 10^{17}-1.89 \times 10^{17}$ and 1.29 $\times 10^{17}-1.78 \times 10^{17} \mathrm{~cm}^{-3}$, for $500 \mathrm{~mJ}$ and $450 \mathrm{~mJ}$ energy respectively. The graph of the electron temperature against the distance along the plasma expansion revealed that, the plasma expansion rapidly drops at larger distance away from the target surface. This was attributed to the radiative recombination and the Bremsstrahlung emission. From the emission spectra of the $\mathrm{Mg}$ lines, we observed a decrease in the line intensities with increasing delay time and also a decrease in both the electron temperature and number density along the distance of the plasma expansion.

\section{Acknowledgement}

This work was supported by National Natural Science Foundation of China (Nos. 61505071, 51405181, 51405200), the Natural Science Foundation of Jiangsu Province (Nos. BK20130407, BK20150526), and the Advanced Talents Foundation of Jiangsu University (Nos. 15JDG036 and 15JDG037).

\section{References}

1. Klein S, Hildenhagen J, Dickmann K, Stratoudaki T, Zafiropulos V (2000) LIBS Spectroscopy for Monitoring and Control of the Laser Cleaning Process of Stone and Medieval Glass. J Cult Herit 1: S287-S292.

2. Martin MZ, Labbé N, Rials TG, Wullschleger SD (2005) Analysis of PreservativeTreated Wood by Multivariate Analysis of Laser-Induced Breakdown Spectroscopy Spectra. Spectrochim Acta Part B At Spectrosc 60: 1179-1185.

3. Samek O, Beddows D, Telle H, Kaiser J, Liška M, et al. (2001) Quantitative laser-induced breakdown spectroscopy analysis of calcified tissue samples Spectrochim Acta Part B At Spectrosc 56: 865-875.

4. Carmona N, Oujja M, Rebollar E, Römich H, Castillejo M (2005) Analysis of corroded glasses by laser induced breakdown spectroscopy. Spectrochim Acta Part B At Spectrosc 60: 1155-1162.

5. Barrette L, Turmel S (2001) On-Line Iron-Ore Slurry Monitoring for Real-Time Process Control of Pellet Making Processes Using Laser-Induced Breakdown 
Citation: Hongbing Y, Asamoah E, Jiawei C, Dongqing Y, Fengxiao Y (2018) Comprehensive Study on the Electron Temperature and Electron Density of Laser-Induced Mg Plasma. J Laser Opt Photonics 5: 181. doi: 10.4172/2469-410X.1000181

Page 6 of 6

Spectroscopy: Graphitic vs. Total Carbon Detection. Spectrochim Acta Part B At Spectrosc 56: 715-723.

6. Gornushkin I, Kazakov AY, Omenetto N, Smith B, Winefordner J (2005) Experimental verification of a radiative model of laser-induced plasma expanding into vacuum. Spectrochim Acta Part B At Spectrosc 60: 215-230.

7. Barthélemy O, Margot J, Chaker M, Sabsabi M, Vidal F, et al. (2005) Influence of the laser parameters on the space and time characteristics of an aluminum laser-induced plasma Spectrochim Acta Part B At Spectrosc 60: 905-914.

8. Sabsabi M, Cielo P (1995) Quantitative Analysis of Aluminum Alloys by Laser-Induced Breakdown Spectroscopy and Plasma Characterization. Appl Spectrosc 49: 499-507.

9. Omenetto N, Hahn DW (2015) Laser-Induced Breakdown Spectroscopy (LIBS), Part II: Review of Instrumental and Methodological Approaches to Material Analysis and Applications to Different Fields. Spectrochim Acta Part B At Spectrosc 66: 347-419.

10. Corsi M, Cristoforetti G, Giuffrida M, Hidalgo M, Legnaioli S, et al. (2004) Threedimensional analysis of laser induced plasmas in single and double pulse configuration Authors. Spectrochim Acta Part B At Spectrosc 60: 870-875.

11. Ni ZB, Chen XL, Fu HB, Wang JG, Dong FZ (2014) Study on quantitative analysis of slag based on spectral normalization of laser-induced plasma image. Front Phys 9: 419-438.

12. Cremers DA, Radziemski LJ (2006) Handbook of Laser-Induced Breakdown Spectroscopy. (2 edn.), pp: 23-52.

13. Miziolek AW, Palleschi V, Schechter I (2006) Laser-Induced Breakdown Spectroscopy (LIBS): Fundamentals and Applications. Cambridge University Press.

14. Zhang S, Wang X, He M, Jiang Y, Zhang B, et al. (2014) Laser-induced plasma temperature. Spectrochim Acta Part B At Spectrosc 97: 13-33.

15. Stavropoulos P, Palagas C, Angelopoulos G, Papamantellos D, Couris S (2004) Calibration Measurements in laser-induced breakdown spectroscopy using nanosecond and picosecond lasers. Spectrochim Acta Part B At Spectrosc 9 : 1885-1892.

16. Asamoah E, Hongbing $Y$ (2016) Influence of laser energy on the electron temperature of a laser-induced Mg plasmaApplied Physics B 123: 22.

17. Krähling T, Geisler S, Okruss M, Florek S, Franzke J (2015) Spectroscopic measurements of the electron number density, electron temperature and $\mathrm{OH}(\mathrm{A})$ rotational distribution in a liquid electrode dielectric barrier discharge. Spectrochimica Acta Part B: Atomic Spectroscopy 114: 20-26.

18. Mahmood S, Shaikh NM, Kalyar MA, Rafiq M, Piracha NK, et al. (2009) Measurements of electron density, temperature and photoionization cross sections of the excited states of neon in a discharge plasma. Journal of Quantitative Spectroscopy and Radiative Transfer 110: 1840-1850.

19. Camacho JJ, Diaz L, Martinez-Ramirez S, Caceres JO (2015) Time- and space-resolved spectroscopic characterization of laser-induced swine muscle tissue plasma. Spectrochimica Acta Part B: Atomic Spectroscopy 111: 92-101.

20. Moon HY, Smith BW, Omenetto N, Winefordner JD (2012) On the usefulness of a duplicating mirror to evaluate self-absorption effects in laser induced breakdown spectroscopy. Chemical Physics 398: 221-227.

21. Shoursheini SZ, Sajad B, Parvin P (2010) Determination of gold fineness by laser induced breakdown spectroscopy with the simultaneous use of $\mathrm{CW}-\mathrm{CO} 2$ and Q-SW Nd:YAG lasers. Optics and Lasers in Engineering 48: 89-95.

22. Mortazavi SZ, Parvin P, Mousavi Pour MR, Reyhani A, Moosakhani A, et al. (2014) Time-resolved evolution of metal plasma induced by $Q$-switched Nd:YAG and ArF-excimer lasers. Optics \& Laser Technology 62: 32-39.

23. Cristoforetti G, Giacomo ADe, Dell'Aglio M, Legnaioli S, Tognoni E, Pallesch V, et al. (2010) Local Thermodynamic Equilibrium in Laser-Induced Breakdown Spectroscopy: Beyond the McWhirter criterion. Spectrochimica Acta Part B: Atomic Spectroscopy 65: 86-95

24. Hafeez S, Shaikh NM, Rashid B, Baig MA (2008) Plasma properties of laserablated strontium target Journal of Applied Physics 103: 083-117.

25. Aguilera JA, Aragón C (2004) Characterization of a laser-induced plasma by spatially resolved spectroscopy of neutral atom and ion emissions.: Comparison of local and spatially integrated measurements. Spectrochimica Acta Part B: Atomic Spectroscopy 59: 1861-1876.

26. http://Physics.nist.gov/PhysRefData/ASD/lines_form.html

27. Gornushkin IB, King LA Smith BW, Omenetto N, Winefordner JD (1999) Line broadening mechanisms in the low pressure laser-induced plasma Spectrochimica Acta Part B: Atomic Spectroscopy 54: 1207-1217.

28. Shaikh NM, Rashid B, Hafeez S, Jamil Y, Baig M (2006) Measurement of electron density and temperature of a laser-induced zinc plasma. Journal of Physics D: Applied Physics 39: 1384 